\title{
Glutathione-S-Transferase Subtypes $\alpha$ and $\pi$ as a Tool to Predict and Monitor Graft Failure or Regeneration in a Pilot STUdy OF Living DONOR Liver Transplantation
}

\author{
C. Jochum ${ }^{1}$, M. Beste ${ }^{1}$, J.-P. Sowa ${ }^{1}$, M. S. Farahani1 ${ }^{1}$, V. Penndorf ${ }^{1}$, S. Nadalin ${ }^{2}$, F. Saner ${ }^{3}$, A. Canbay ${ }^{1}$, \\ G. Gerken ${ }^{1}$ \\ ${ }^{1}$ Clinic of Gastroenterology and Hepatology, University Hospital Essen, Essen, Germany, \\ ${ }^{2}$ Division of Transplant Surgery, Eberhard Karls University, Tübingen, Germany, \\ ${ }^{3}$ Clinic of General and Transplantation Surgery, University Hospital Essen, Essen, Germany
}

\begin{abstract}
Objective: Glutathione-S-Transferase (GST) subtype $\alpha$ and $\pi$ are differentially expressed in adult liver tissue. Objective of the study was if GST $\alpha$ and $\pi$ may serve as predictive markers for liver surgery, especially transplantations.

Methods: 13 patients receiving living donor liver transplantation (LDLT) and their corresponding donors were analyzed for standard serum parameters (ALT, AST, $\gamma$ GT, bilirubin) as well as GST- $\alpha$ and $-\pi$ before LDLT and daily for 10 days after LDLT. Patients (R) and donors (D) were grouped according to graft loss (R1/D1) or positive outcome (R2/D2) and above named serum parameters were compared between the groups.

Results: R1 showed significantly increased GST- $\alpha$ and significantly lower GST- $\pi$ levels than R2 patients or the donors. There was a positive correlation between GST- $\alpha$ and ALT, AST as well as bilirubin and a negative correlation to $\gamma$ GT. However, $\gamma$ GT correlated positively with GST- $\pi$. Graft failure was associated with combined low GST- $\pi$ levels in donors and their recipients before living donor liver transplantation.

Conclusion: Our data suggest that high GST- $\alpha$ serum levels reflect ongoing liver damage while GST- $\pi$ indicates the capacity and process of liver regeneration. Additionally, GST- $\pi$ may be useful as marker for optimizing donor and recipient pairs in living donor liver transplantation.
\end{abstract}

Key words: living donor liver transplantation, liver regeneration, glutathione-S-transferase $\alpha$ and $\pi$, cholestasis

Abbreviations: D, donor group; D1, donors corresponding to recipients with liver failure; D2, donors corresponding to recipients with positive outcome; GST- $\alpha$, glutathione-S-transferase-alpha; GST- $\pi$, glutathione-S-transferase-pi; LDLT, Living Donor Liver Transplantation; POD, post operative day; $\mathrm{R}$, recipient group; R1, recipients with liver failure; R2, recipients with positive outcome.

\section{INTRODUCTION}

Living donor liver transplantation (LDLT) has become an alternative to deceased donor liver transplantation in the western countries to overcome the perpetual shortage of donor organs. The unique ability of the liver to regenerate completely after resection makes this approach possible. Remarkably, the liver volume restoration occurs within four weeks after LDLT in the vast majority of both donors and recipients $[1,2$, $3-5]$. However, in some cases the transplanted liver exhibits impaired regeneration, which consequently leads to liver failure [6]. Therefore, it remains a challenge to identify those livers with and without sufficient capacity to regenerate after LDLT or partial hepatectomy.

Recently, it has been shown in a rat model of cirrhosis and regeneration that the enzyme glutathione-stransferase (GST) and their predominant hepatic subtypes show a distinct pattern during regeneration [7]. In general, GST comprises various subtypes of enzymes, catalyzing the binding of glutathione to xenobiotics, thus facilitating their detoxification. Up to $3 \%$ of soluble protein in adult hepatocytes constitutes of GST subtype alpha (GST- $\alpha$ ), while GST subtype pi (GST- $\pi$ ) is expressed in fetal hepatocytes and in the placenta [8]. Expression of GST- $\pi$ in adult liver tissue is restricted to bile duct cells and constitutes a phenotypic marker of oval cells [9]. Oval cells represent a bipotent population of progenitor cells, which can differentiate into hepatocytes and biliary epithelial cells $[10,11]$. These cells are involved in liver regeneration following severe liver injury and in liver carcinogenesis $[12,9]$. Different studies in animal models of partial hepatectomy identified GST- $\pi$ expression in oval cells as relevant for liver regeneration $[13,14,15]$. However, the regulation of oval cell recruitment and differentiation as well as their precise role remains unclear.

GST enzymes are intracellular cytosolic enzymes. Thus, GST serum levels rather than protein expression reflect cell injury or cell turnover. The half-life of GST is short (<90 min.) (6), therefore changes in GST-levels closely match ongoing liver cell death. In- 
deed, in acute liver failure GST- $\alpha$ is highly elevated and is associated with worse outcome (unpublished data). GST- $\alpha$ levels are also significantly elevated in liver transplant recipients who develop moderate or severe rejection after liver transplantation compared to those recipients with mild or no rejection [16]. In contrast, GST- $\pi$ levels remain stable even in cases of severe rejection, suggesting both enzymes are restricted to different cell types and play independent roles in organ rejection [16] . Apart from this high expression levels of GST- $\pi$ were seen in patients with liver cirrhosis and chronic hepatitis [17].

The aim of our study was to determine whether serum GST- $\alpha$ and GST- $\pi$ levels are related to liver regeneration after LDLT in donors and recipients. To check this we proposed several questions: i) Are GST$\alpha$ and GST- $\pi$ differentially released into the serum in donors and recipients before or after LDLT? ii) Are increased GST- $\alpha$ levels associated with transplant loss? And finally (iii) are lower GST- $\pi$ levels associated with impaired liver regeneration?

To study these questions donors and recipients in LDLT were compared for GST serum levels and outcome. Furthermore donors and recipients each were split into two sub-groups, donors and corresponding recipients with transplant loss, due to vascular complications, occurring in the early postoperative period (4 weeks) were subsequently labeled as D1 and R1. Recipients who recovered uneventfully and corresponding donors were labeled as R2 and D2, respectively.

\section{MAterial And Methods}

\section{Characteristics of Patients AND DONORS}

In this study 13 consecutive living donors of a right liver lobe (segment 5-8) and their recipients were included. All patients provided informed consent. All donors were healthy and eligible for LDLT following the guidelines for selection of living related liver donors at the University of Essen (Germany). Demographics of donors and recipients as well as the indications for LDLT are summarized in Table 1. The recipients received between 54.7 and $66.4 \%$ of the donor's liver volume (median: 60.6\% mean: 60.4\%). Four recipients required retransplantation due to organ failure within the first 4 weeks after transplantation, thus con- stituting group R1 with the respective donor group D1. Groups R2 and D2 included the remaining 9 recipients and donors with positive outcome.

\section{LABORATORY DATA}

Serum and plasma samples of donors and recipients were obtained as a baseline (POD 0) three days to six weeks prior to the transplantation. Serum and plasma samples were obtained daily $(7.30 \mathrm{am} \pm 15 \mathrm{~min})$ from POD 1 to 10 . The plasma samples were immediately stored on ice, centrifuged and stored at $-20^{\circ} \mathrm{C}$. Bilirubin, AST, ALT and $\gamma$ GT were determined at the Central Laboratory of the University of Essen.

The concentrations of GST- $\alpha$ and GST- $\pi$ were measured using commercially available ELISA-Kits (Biotrin International GmbH, Dreieich, Germany). The concentrations of GST- $\alpha$ were determined in serum and concentrations of GST- $\pi$ in plasma. The internal controls were within the sensitivity of the test.

\section{Data Analyses and Statistics}

All data are shown as mean \pm SEM, if not stated otherwise. The data were analyzed for day to day changes and for the time course after LDLT. For statistical analyses a two-sided t-test for unrelated groups was used. Statistical significance was assumed for $\mathrm{p}<0.05$. Calculations were done using GraphPad Prism' Software. Correlations were calculated using SPSS Software, employing non-parametric tests (Spearman rank correlation).

\section{RESULTS}

There are no significant differences in GST- $\alpha$ levels between donors and recipients before LDLT. Previous to LDLT (POD 0) higher average values of GST- $\alpha$ were found in donors than in recipients (6.4 vs. $4.98 \mu \mathrm{g} / \mathrm{ml})$. Both remained in the normal range of $<7.2 \mu \mathrm{g} / \mathrm{ml}$ and the differences were not significant $p$ $=0.37$ (Fig. 1A). Additionally neither R1 vs. R2 nor D1 vs. D2 showed any differences at POD 0.

Graft loss is associated with higher GST- $\alpha$ level. At POD 1, the mean serum levels of GST- $\alpha$ increased significantly in both donors and recipients

Table 1. Patient characteristics: Two recipients with toxic cirrhosis were also infected with Hepatitis $\mathrm{C}$ virus. 6 recipients had a HCC in the cirrhotic liver. (Abbr.: HCC= hepatocellular carcinoma; PSC= primarily sclerosing cholangitis).

\begin{tabular}{ccc}
\hline Parameter & Donors & Recipients \\
\hline Age & $23-59$ ys; median 30ys & 26-65ys; median 50ys \\
Male & 7 & 8 \\
Female & 6 & 5 \\
(Ethyl-)toxic Cirrhosis & - & 6 \\
Hepatitis C & - & 2 \\
Hepatitis B & - & 2 \\
HCC & - & 6 \\
PSC & - & 1 \\
Autoimmune Hepatitis & - & 3 \\
Cryptogenic Cirrhosis & - &
\end{tabular}


A

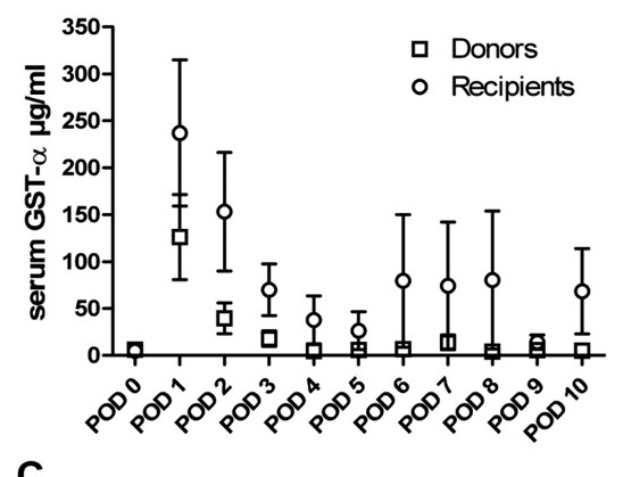

C

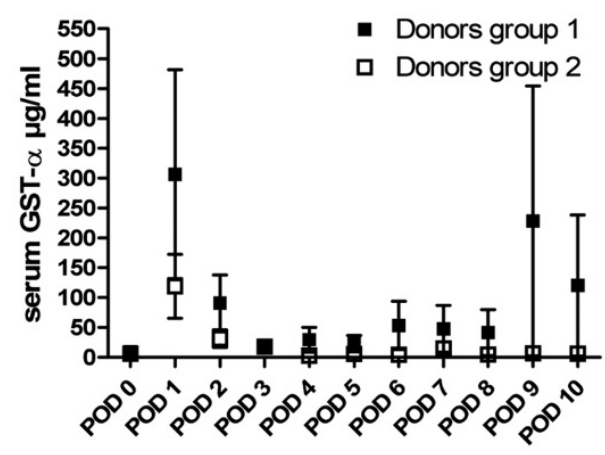

B

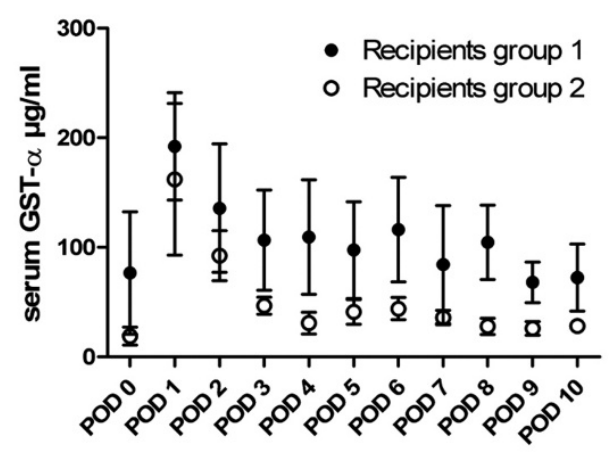

Fig. 1. GST- $\alpha$ in Donors and Recipients. Shown are average serum GST- $\alpha$ values with SEM during 10 days after transplantation, with a single pre operative sample as reference (POD 0). A: Time course of all donors and recipients. B: D1 = Donors of patients with graft loss; D2 = Donors of patients with beneficial outcome. C: R1 = recipients who suffered from graft loss within 4 weeks after LDLT.; R2 = recipients with positive outcome.

$(p=0.02)$. This elevation of GST- $\alpha$ levels was stronger in recipients during the post-transplantation period compared to donors $(p=0.04)$. Over the following days GST- $\alpha$ serum values decreased in donors and recipients, reaching preoperative values in donors at POD 4 and in recipients at POD 5. R1 patients exhibited significantly higher serum levels of GST- $\alpha$ than the R2 group ( $p=0.002$; Fig. 1B) and all donors $(p=0.003)$ in the observed post transplantation phase. In contrast the time course in $\mathrm{R} 2$ patients compared to all donors did not display significant differences and GST- $\alpha$ levels reached preoperative values after POD 4 as did both donor groups.

Lower plasma GST- $\pi$ in donors and recipients before LDLT is associated with graft loss. At POD 0 donor and recipient GST- $\pi$ levels were on average quite similar (donors: $65.4 \mathrm{ng} / \mathrm{ml}$; recipients: $67.4 \mathrm{ng} / \mathrm{ml}$; Fig. 2A), on the contrary subgroup analysis demonstrated differences. R1- and D1-individuals showed significantly lower GST- $\pi$ values than R2- and D2-individuals at POD 0 (Fig. 2B, C). Analysis of the individual donor-recipient pairs revealed GST- $\pi$ values below $50 \mathrm{ng} / \mathrm{ml}$ in both, donor and corresponding recipient, in pairs where graft loss occurred (Fig. 2D). Conversely, in cases with positive outcome plasma GST- $\pi$ was high in donor and/or recipient. The GST$\pi$ levels increased in all donors after resection and remained elevated through POD 10, although with a broad variance. After transplantation in both recipient groups significantly lower GST- $\pi$ levels were found than in the donors $(p=0.00001)$. Subgroup R1 displayed significantly lower GST- $\pi$ values during the postoperative period compared to $\mathrm{R} 2$ or all donors $\left(p=0.001 ; p=4.7 \times 10^{-8}\right)$. Interestingly, the R2-patients also were found to have significantly lower GST$\pi$ levels than the donors $(p=0.003)$.
There are no significant differences in conventional liver function tests between donors and recipients in the early phase after LDLT. AST and ALT levels were significantly increased on POD 0 in recipients compared to donors (Fig. 3). Donors as well as recipients exhibited a short term increase of AST and ALT after transplantation but both enzymes decreased over time back to normal levels. The observed increase in AST and ALT was more articulate in groups $\mathrm{D} 1$ and R1 in comparison to D2 and R2, respectively, although the differences failed to reach significance.

$\gamma$ GT was significantly elevated in recipients compared to donors on POD 0. From POD 1 to POD 4 $\gamma$ GT in recipients decreased but was still significantly higher than in donors. After POD 5 the serum levels raised in both donors and recipients, though the increase was more prominent in recipients. In D1 and D2 no differences in the serum $\gamma$ GT were found, staying low until POD 4, with a constant rise detectable from POD 5 to POD 10. A similar time course was found in the R2 group, with a raise of serum $\gamma$ GT toward the end of the observation period (POD 5 to 10). R1 patients did not exhibit a comparable pattern, but stayed at low $\gamma$ GT values.

Preoperative bilirubin levels were significantly elevated in recipients referring to donors. Serum bilirubin constantly increased during POD 1 to 4 and regressed to normal levels until POD10. A similar pattern was observable in D1 and D2, although D2 patients had slightly higher serum values. Recipients displayed a reduction of bilirubin in serum on POD 2, slowly returning to raised preoperative levels from POD 3 to POD 8. While R1 bilirubin was significantly reduced compared to R2 on POD 0, no significant differences between these groups were detected after transplantation. 
A

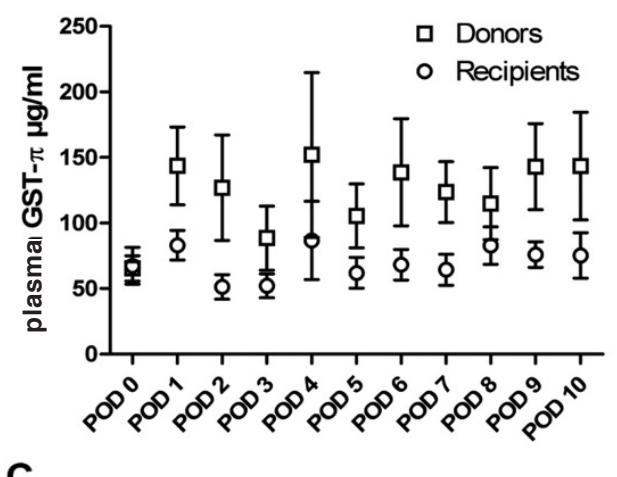

C

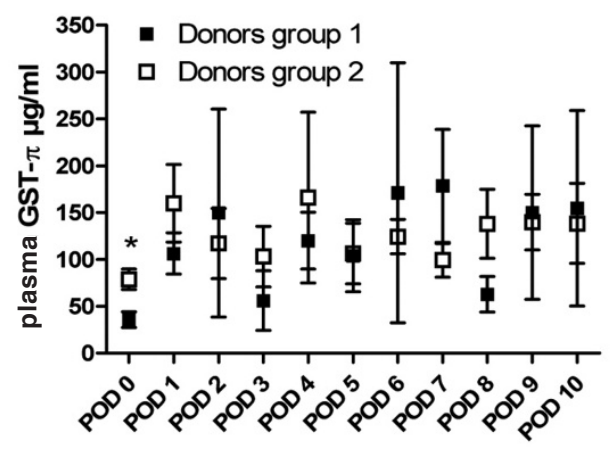

B
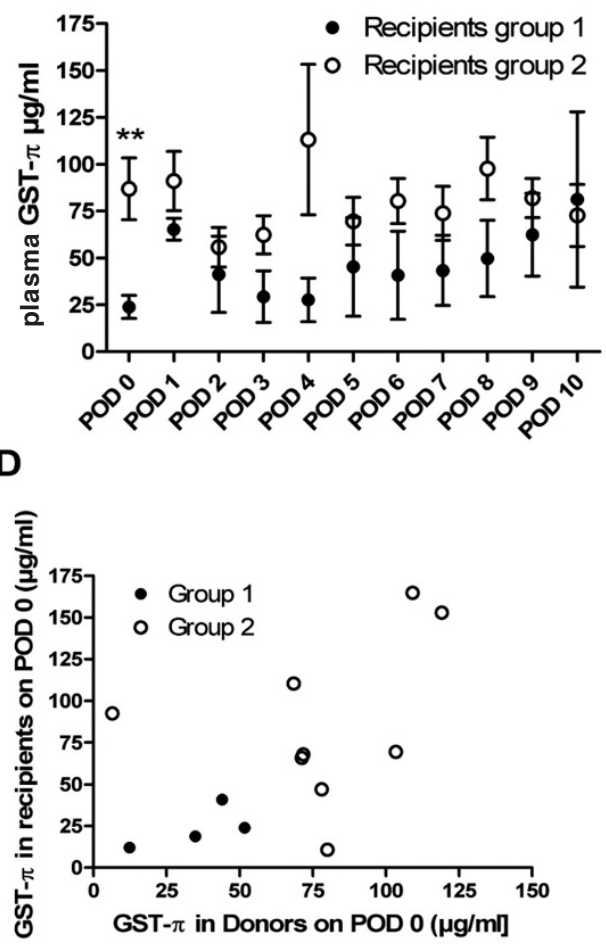

Fig. 2. GST- $\pi$ in Donors and Recipients. Average plasma GST- $\pi$ values with SEM during 10 days after transplantation. As reference a single pre operative time point (POD0) was used. A: Time course of all donors and recipients. B: D1 = Donors of patients with graft loss; D2 = Donors of patients with beneficial outcome. C: R1 = recipients who suffered from graft loss within 4 weeks p.o.; R2 = recipients with positive outcome. D: GST-p values before LDLT of individual donor and recipient pairs. Black dots represent donors and recipients of group 1, white dots those of group 2. *: $p<0.05 ; * * p<0.01$.

A

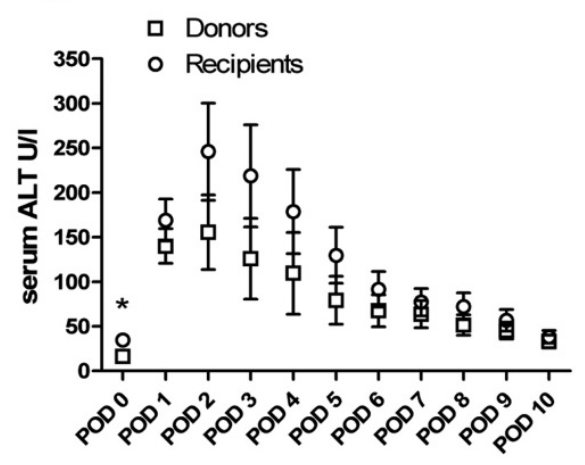

C

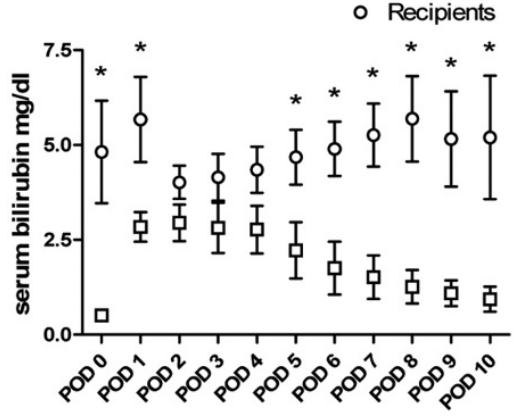

B

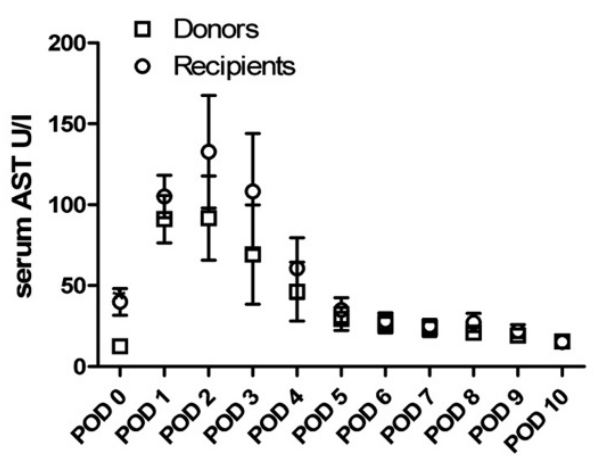

D

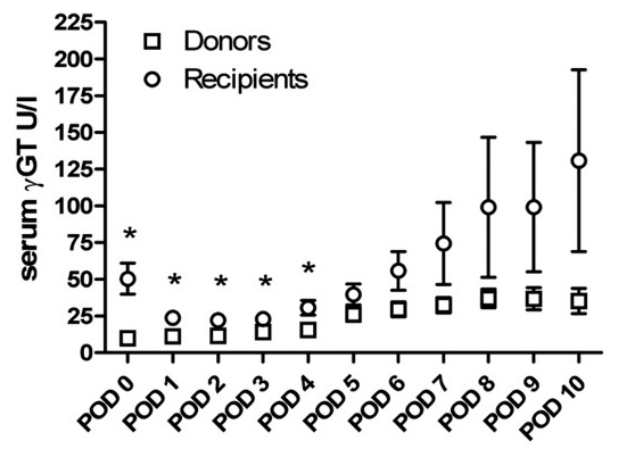

Fig. 3. Liver Function Parameters in Donors and Recipients. Time courses of standard serum parameters for liver damage for donors and recipients and the subgroups D1, D2, R1 and R2. A: ALT; B: AST; C: Bilirubin; D: $\gamma \mathrm{GT} ; *$ : $p<0.05$. 
Table 2. Correlation between Liver Function Parameters and GST for Donors and Recipients. Spearman-Rho correlation coefficients of GST- $\alpha$ and GST- $\pi$ with ALT, AST, $\gamma$ GT and bilirubin as well as p-values of each correlation in donors and recipients.

\begin{tabular}{l|cccc}
\hline & ALT & AST & Bilirubin & \\
\hline & \multicolumn{4}{c}{ Donors } \\
GST- $\boldsymbol{\alpha}$ & $0,378(p<0.001)$ & $0,399(p<0.001)$ & $0,323(p<0.001)$ & $-0.001(p=0.991)$ \\
GST- $\boldsymbol{\pi}$ & $-0.072(p=0.414)$ & $-0.153(p=0.082)$ & $-0.144(p=0.099)$ & $0.212(p=0.017)$ \\
\hline \multirow{2}{*}{ GST- $\boldsymbol{\alpha}$} & $0.597(p<0.001)$ & $0.629(p<0.001)$ & $0.315(p<0.001)$ & $-0.330(p<0.001)$ \\
GST- $\boldsymbol{\pi}$ & $0.69(p=0.425)$ & $0.114(p=0.188)$ & $0.05(p=0.563)$ & $0.307(p<0.001)$ \\
\hline
\end{tabular}

Serum $\gamma$ GT correlates positively with GST- $\pi$ and negatively with GST- $\alpha$. Non-parametric correlation analyses of GST- $\alpha$ and GST- $\pi$, respectively, with the established serum parameters AST, ALT, $\gamma \mathrm{GT}$ and bilirubin were conducted for donors and recipients (Table 2). GST- $\pi$ correlated significantly positive with $\gamma$ GT in recipients and donors (R: 0.307, $p<0.001$; $\mathrm{D}: 0.212, \mathrm{p}=0.017)$. In contrast, GST- $\alpha$ was significantly negatively correlated with $\gamma \mathrm{GT}$ in recipients ($0.33, p<0.001)$ and positively correlated with ALT, AST and bilirubin in recipients and donors.

\section{Discussion}

Liver regeneration in different settings, for example in partial hepatectomy, acute liver failure, and in living related liver transplantation (LDLT), respectively, is a major issue in hepatology. Despite quick recovery of liver volume [5] qualitative regeneration takes longer and might influence the outcome of $\operatorname{LDLT}[4,18]$ Predictive markers, obtainable in a non invasive fashion, are warranted to estimate chances of patients to appropriately regenerate after liver surgery. Patient survival on the one hand and liver failure after transplantation on the other hand could be improved by reliable indicators of expected regeneration.

Glutathione-s-transferase (GST) might serve as an early marker in liver regeneration. Indeed, GST- $\alpha$ levels peaked at POD 1 both in donors and recipients with a decline over the following days. Highest GST- $\alpha$ values were found in those recipients who lost their grafts (R1) during the postoperative period, suggesting increased hepatocyte death and liver function impairment. Serum levels in R1-patients were significantly elevated compared to donors and the R2-group. Of note, there were no significant differences in GST- $\alpha$ levels between donors and R2-individuals. These findings suggest increased GST- $\alpha$ as a possibly useful indicator for poor outcome and eventual graft loss and are consistent with data from deceased donor liver transplantations [16]. Additionally, GST- $\alpha$ showed positive correlation to ALT and AST and bilirubin, which could imply a connection between liver damage in general and GST- $\alpha$ release into serum. In vitro data demonstrated that the expression of GST- $\alpha$ increases in response to different drugs, though, the influence of immunosuppressive agents on GST- $\alpha$ expression has not been tested so far [19]. However, in our study GST- $\alpha$ levels of the recipients with good outcome were similar to those of donors, who did not receive immunosuppressive drugs. Taken together, high GST$\alpha$ values in recipients with poor outcomes seem to reflect a higher cell death with impaired liver function.

It is well known that GST- $\pi$ is mainly expressed in bile ducts and in oval cells [20] which partially constitute the regenerative capacity of the liver [21]. Usually, raised serum levels of cholestatic enzymes are deemed as markers for advanced fatty liver disease [22] or damage to bile duct cells in chronic viral hepatitis [23]. But as we have previously shown in patients with acute liver failure, $\gamma$ GT is significantly increased in patients with positive outcome after acute liver failure [24]. Massive cell injury in the liver is known to trigger ductular proliferation involving mature cholangiocytes and hepatocytes $[25,26]$. It was also shown that an increase in bile acids after partial hepatectomy is indicative of liver regeneration $[27,28]$. Thus, cholestatic enzymes (such as $\gamma$ GT) may increase as a consequence of elevated bile acids that are associated with proliferation in the course of liver regeneration. In our patient cohort we found a significant correlation between GST- $\pi$ and $\gamma$ GT. Regarding the overall picture of GST- $\pi$ and cholestatic enzymes as $\gamma$ GT a fast and short increase of these parameters in patient sera may imply regenerative activity and thus a positive outcome for surgical therapy. In contrast, continuously raised serum levels still could be indicative for ongoing long term liver damage and/or for a constant insufficient regeneration. Graft survival was associated with higher GST- $\pi$ values in recipients. The R1 recipients exhibited significantly lower plasma levels, below $50 \mu \mathrm{g} / \mathrm{ml}$. D1-patients were also found to have significantly lower GST- $\pi$ values before liver resection. The analysis of individual donor/recipient pairs revealed that low GST- $\pi$ levels in donors as well as corresponding recipients at POD 0 were associated with raised serum ALT and AST and graft failure within 4 weeks postoperatively. In contrast to this no graft loss occurred with GST- $\pi$ levels above $50 \mu \mathrm{g} / \mathrm{ml}$ in either donor or recipient.

The significantly lower GST- $\pi$ levels before LDLT in the donor-recipient pairs, whose transplant failed, indicate a link between this marker and graft failure. However, GST- $\pi$ values of the D1 group increased at 
an adequate rate and no significant differences to D2 were detectable during the post transplant period. Strikingly, the aforementioned postoperative GST- $\pi$ increase did not occur in the R1 group.

Taken together, these observations suggest that higher levels of GST- $\pi$ reflect normal liver regeneration. Since no correlation to bilirubin could be observed, GST- $\pi$ may rather indicate a higher turnover of oval cells and bile duct proliferation during liver regeneration than cholestatic damage. From the present data it is not yet possible to say if low GST- $\pi$ may have a causal connection to the early graft loss or if the low values are an additional consequence of liver cell damage leading to graft failure. Some causal connection is supported by the observation that only recipients whose donors exhibited simultaneously low pretransplant GST- $\pi$ values developed graft failure. Since the numbers of patients in our study is low and comparable animal studies are lacking, this remains speculative.

Nevertheless, our data indicate that higher GST- $\pi$ levels predict a favorable outcome and support an important role of oval cells and bile duct proliferation during the process of liver regeneration in humans after LDLT. Furthermore, our data suggest that low GST$\pi$ levels in donors and their recipients implicate a higher risk for graft failure and could therefore denote a preoperative negative indicator for patients on waiting list for deceased donor transplantation or in preparation for LDLT. Further analysis of additional donor and recipient pairs in LDLT is warranted to specify limiting values for plasma GST- $\pi$ before and after transplantation.

In conclusion the data at hand indicate a role for GST- $\alpha$ in ongoing liver damage while GST- $\pi$ seems representative for regenerative capacity. Both parameters could may be a complementary way to describe or even predict the possible outcome of LDLT.

Grants: This work was supported by the Deutsche Forschungsgemeinschaft (DFG, grant 267/4-1 and 267/6-1), and the Wilhelm Laupitz Foundation to AC. Additional support from Biotrin International GmbH (Dreieich, Germany)

Conflict of interest: None of the authors has to report any conflict of interest regarding this work.

\section{REFERENCES}

1. Emre S. Living donor liver transplantation: a critical review. TransplantProc. 2001 11;33(7-8):3456-7.

2. Liu LU, Schiano TD. Adult live donor liver transplantation. Clinics in liver disease. 2005 Nov;9(4):767-86, viii-ix.

3. Liu CL, Fan ST. Adult-to-adult live-donor liver transplantation: the current status. Journal of hepato-biliary-pancreatic surgery. 2006;13(2):110-6.

4. Jochum C, Beste M, Penndorf V et al. Quantitative liver function tests in donors and recipients of living donor liver transplantation. Liver Transpl. 2006 Apr;12(4):544-9.

5. Nadalin S, Malago M, Testa $G$ et al. Volume increase and functional recovery in rest liver and graft after living donor liver transplantation in adults. Hepatology. 2001;34 Pt2:200a.

6. Pan GD, Yan LN. Problems in adult living donor liver transplantation using the right hepatic lobe. Hepatobiliary Pancreat Dis Int. 2006 Aug;5(3):345-9.
7. Liu EH, Chen MF, Yeh TS et al. A useful model to audit liver resolution from cirrhosis in rats using functional proteomics. The Journal of surgical research. 2007 Apr; 138(2):214-23.

8. Hiley C, Fryer A, Bell J, Hume R, Strange RC. The human glutathione S-transferases. Immunohistochemical studies of the developmental expression of Alpha- and Pi-class isoenzymes in liver. BiochemJ. 1988 08/15/; 254(1):255-9.

9. Lowes KN, Brennan BA, Yeoh GC, Olynyk JK. Oval cell numbers in human chronic liver diseases are directly related to disease severity. AmJPathol. 1999 02;154(2):53741.

10. Lemire JM, Shiojiri N, Fausto N. Oval cell proliferation and the origin of small hepatocytes in liver injury induced by D-galactosamine. AmJPathol. 1991 09;139(3):535-52.

11. Hixson DC, Chapman L, McBride A, Faris R, Yang L. Antigenic phenotypes common to rat oval cells, primary hepatocellular carcinomas and developing bile ducts. Carcinogenesis. 1997 06;18(6):1169-75.

12. Sell S, Dunsford HA. Evidence for the stem cell origin of hepatocellular carcinoma and cholangiocarcinoma. AmJ Pathol. 1989 06;134(6):1347-63.

13. Evarts RP, Nakatsukasa H, Marsden ER, Hsia CC, Dunsford HA, Thorgeirsson SS. Cellular and molecular changes in the early stages of chemical hepatocarcinogenesis in the rat. Cancer Res. 1990 06/01/;50(11):3439-44.

14. Isfort RJ, Cody DB, Stuard SB et al. The combination of epidermal growth factor and transforming growth factorbeta induces novel phenotypic changes in mouse liver stem cell lines. JCell Sci. 1997 12;110 (Pt 24):3117-29.

15. Lowes KN, Croager EJ, Olynyk JK, Abraham LJ, Yeoh GC. Oval cell-mediated liver regeneration: Role of cytokines and growth factors. JGastroenterolHepatol. 2003 01;18(1):4-12.

16. Dickson RC, Lauwers GY, Rosen CB, Cantwell R, Nelson DR, Lau JY. The utility of noninvasive serologic markers in the management of early allograft rejection in liver transplantation recipients. Transplantation. 1999 07/27/;68(2):247-53.

17. Tsuchida S, Sekine Y, Shineha R, Nishihira T, Sato K. Elevation of the placental glutathione S-transferase form (GST-pi) in tumor tissues and the levels in sera of patients with cancer. Cancer Res. 1989 09/15/;49(18): 5225-9.

18. Nadalin S, Testa G, Malago M et al. Volumetric and functional recovery of the liver after right hepatectomy for living donation. Liver Transpl. 2004;10(8):1024-9.

19. Morel F, Fardel O, Meyer DJ et al. Preferential increase of glutathione S-transferase class alpha transcripts in cultured human hepatocytes by phenobarbital, 3-methylcholanthrene, and dithiolethiones. Cancer Res. 1993 01/15/;53(2):231-4.

20. Ma X, Qiu DK, Peng YS. Immunohistochemical study of hepatic oval cells in human chronic viral hepatitis. World J Gastroenterol. 2001 Apr;7(2):238-42.

21. Chen YK, Zhao XX, Li JG, Lang S, Wang YM. Ductular proliferation in liver tissues with severe chronic hepatitis B: an immunohistochemical study. World J Gastroenterol. 2006 Mar 7;12(9):1443-6.

22. Benini F, Pigozzi MG, Baisini $O$ et al. Increased serum gamma-glutamyl-transpeptidase concentration is associated with nonalcoholic steatosis and not with cholestasis in patients with chronic hepatitis C. Journal of gastroenterology and hepatology. 2007 Oct;22(10):1621-6.

23. Giannini E, Botta F, Fasoli A et al. Increased levels of gammaGT suggest the presence of bile duct lesions in patients with chronic hepatitis $\mathrm{C}$ : absence of influence of HCV genotype, HCV-RNA serum levels, and HGV infection on this histological damage. Digestive diseases and sciences. 2001 Mar;46(3):524-9. 
24. Canbay A, Jochum C, Bechmann LP et al. Acute liver failure in a metropolitan area in Germany: a retrospective study (2002-2008). Z Gastroenterologie. 2009 Sep;47(9): 807-13.

25. Demetris AJ, Seaberg EC, Wennerberg A, Ionellie J, Michalopoulos G. Ductular reaction after submassive necrosis in humans. Special emphasis on analysis of ductular hepatocytes. Am J Pathol. 1996 Aug;149(2):439-48.

26. Fausto N, Campbell JS, Riehle KJ. Liver regeneration. Hepatology. 2006 Feb;43(2 Suppl 1):S45-53.

27. Huang W, Ma K, Zhang J et al. Nuclear receptor-dependent bile acid signaling is required for normal liver regeneration. Science. 2006 Apr 14;312(5771):233-6.

28. Geier A, Trautwein C. Bile acids are "homeotrophic" sensors of the functional hepatic capacity and regulate adaptive growth during liver regeneration. Hepatology. 2007 Jan;45(1):251-3.
Received: January 13, 2010 / Accepted: March 18, 2010

Address for correspondence:

Guido Gerken MD

University Hospital Essen

Hufelandstr. 55

45122 Essen

Germany

Phone: +49 (201) 723-3610

Fax: $\quad+49$ (201) 723-5971

E-mail: guido.gerken@uk-essen.de 